\title{
PRÁCTICAS DE LECTURA Y ESCRITURA EN EDUCACIÓN SUPERIOR: LO QUE LOS ESTUDIANTES Y DOCENTES DICEN DE LA LECTURA Y LA ESCRITURA EN LAS LICENCIATURAS DE UNA UNIVERSIDAD EN EL CARIBE COLOMBIANO
}

\author{
LITERACY PRACTICES IN HIGHER EDUCATION: \\ WHAT STUDENTS AND TEACHERS SAY READING AND WRITING IN A UNIVERSITY \\ DEGREE IN THE COLOMBIAN CARIBBEAN
}

\author{
Luis Manuel Cárdenas Cárdenas*
}

\begin{abstract}
RESUMEN
El presente artículo es producto del resultado de una investigación realizada en 10 licenciaturas de una Universidad del Caribe colombiano cuyo propósito general fue el de describir, interpretar y comprender las prácticas de lectura y escritura que se llevan a cabo en esas licenciaturas. Para la producción de este artículo se tuvo en cuenta un aspecto que en la investigación en general fue concebido como el primer objetivo específico. Tal aspecto hace referencia a las concepciones que tienen los docentes y estudiantes de las licenciaturas acerca de la enseñanza y aprendizaje de la lectura y escritura de textos académicos en la universidad. El estudio en general toma como fundamento teórico el concepto de alfabetización académica. Los datos se recolectaron a través de observaciones de clases. Se aplicaron encuestas y entrevistas a estudiantes y a profesores de las licenciaturas. Los resultados de la investigación determinaron que el proceso de enseñanza y aprendizaje de la lectura y la escritura en el aula de las licenciaturas es muy limitado. El desarrollo pedagógico y didáctico de las clases carece de prácticas lectoras y escritoras.
\end{abstract}

Palabras clave: Lectura, escritura, prácticas de lectura y escritura, etnografía.

\begin{abstract}
This article is the result of a survey conducted in 10 degrees in a University Colombian Caribbean whose general purpose was to describe, interpret and understand the literacy practices that take place in these degrees. To achieve this article was taken into account one aspect that research in general was conceived as the first specific objective. This aspect refers to the conceptions which teachers and students of the degrees on the teaching and learning of reading and writing academic texts in
\end{abstract}

Fecha de recepción: Marzo 31 de 2014 / Fecha de aceptación: Julio 10 de 2014

Tipología: Artículo de Investigación Científica y Tecnológica

Para citar este artículo: Cárdenas, C. L. (2014). Prácticas de lectura y escritura en educación superior: lo que los estudiantes y docentes dicen de la lectura y la escritura en las licenciaturas de una universidad en el caribe Colombiano, Praxis, Vol. 10, 60 - 77

*Doctor en Educación Universidad del Atlántico, Colombia.E-mail: lic.Icardenas@gmail.com. 
college. The study usually takes as a theoretical concept of academic literacy. Data collected through classroom observations, also came from surveys and interviews with students and teachers of the degrees. The results of the investigation determined that the teaching and learning of reading and writing in the undergraduate classroom is very limited. The development of pedagogical and didactic classes lacks reading and writing practices.

Keywords: Reading, writing, reading and writing practices, ethnography.

\section{INTRODUCCIÓN}

$\mathrm{L}$ a lectura y la escritura de textos académicos y/o científicos es una tarea relevante que requiere esfuerzo, tiempo y práctica, y no ocurre de manera natural (Cassany y Morales, 2008). Quién enseña a comprender y a producir los géneros científicos propios de cada comunidad discursiva es la Universidad. Es en las cátedras universitarias donde se fomenta la producción de textos atendiendo a los códigos de cada especialidad al mismo tiempo que se aprenden los fundamentos de la disciplina, sus objetivos, métodos y procedimientos.

En el contexto universitario, si bien el dominio del contenido es una parte importante, no es única ni determinante. Su uso y comprensión depende, en gran medida, del conocimiento que se tenga de las convenciones propias de la disciplina, donde juega un papel fundamental el dominio de los géneros discursivos que la contemplan. (Cassany y Morales, 2008). De tal manera que estos son esenciales en la vida académica de los estudiantes, pues con ellos aprenden a escribir en diferentes estilos.

En Colombia, el Ministerio de Educación Nacional, ha señalado públicamente la dificultad que tienen los estudiantes universitarios para comprender y escribir textos. Los resultados de las pruebas (Saber PRO) que el ICFES realiza anualmente a los estudiantes universitarios de último semestre continúan reflejando que los estudiantes manifiestan dificultad para interpretar, argumentar y proponer en los textos que leen y escriben. Los resultados que el ICFES presenta son el producto de una evaluación diagnóstica de comprensión lectora en la que sólo evalúa aspectos cognitivos. Las investigaciones realizadas en torno al tema de la lectura y la escritura académica en la universidad han permitido develar que existen factores de tipo pedagógico y didáctico que no permiten un adecuado aprendizaje de la lectura y la escritura de las diversas tipologías textuales que se desarrollan en estos contextos y que las instituciones de educación superior han ignorado por completo. Tales factores tienen que ver con las estrategias y los modos de aprender y de enseñar los contenidos de los textos que los estudiantes leen y escriben en el aula de clases.

En la tradición investigativa colombiana no se visualizan investigaciones que indaguen por las estrategias ni que expliquen y den cuenta de los modos de leer y escribir de los universitarios, es decir, el tipo de demandas de lectura o escritura que se hace en la formación que se imparte en cada universidad, o los dispositivos didácticos o pedagógicos que cada una de éstas configura y promueve y que, explícita o tácitamente, causan ciertas prácticas lectoras y escritoras, ciertos modos de leer y escribir y que, en última instancia, promueven un tipo de cultura académica (Rincón y Gil, 2011, 2013). De igual manera, no se encuentran investigaciones destacables que hayan estudiado las relaciones entre concepciones y prácticas de lectura y escritura universitarias.

En la Región Caribe colombiana, tampoco se visualizan investigaciones que den cuenta de estos aspectos arriba señalados. Los pocos estudios realizados en materia de lectura y escritura, se quedan en el solo informe de los estados y niveles de comprensión en que se encuentran los estudiantes que ingresan a la educación superior. En cuanto a la producción escrita de los estudiantes de esta región se sabe muy poco. Los estudios de producción escrita se han enfocado mucho más en comprender los procesos de 
producción de los textos de tipo narrativo. No se ha hecho un estudio que caracterice la forma cómo leen y escriben los estudiantes universitarios ni mucho menos se ha hecho un análisis del lenguaje que utilizan en la elaboración de los mismos. Asimismo, hacen falta estudios que determinen las formas de enseñanza de la lectura y la escritura por parte de los docentes. El presente artículo da conocer en primera instancia, las concepciones que tienen estudiantes y docentes de las licenciaturas de una universidad del Caribe colombiano acerca del aprendizaje y la enseñanza de la lectura y escritura de textos académicos.

\section{METODOLOGÍA}

El estudio optó por el tipo de investigación cualitativa-etnográfica (Strauss A. \& Corbin J., 1990; Hammersley \& Atkinson, 1994; Rimier, 2012, Martínez, 1997, 2009, 2010) Los datos fueron recogidos por medio de entrevistas y observaciones, técnicas normalmente asociadas con los métodos cualitativos. Sin embargo, los datos se codificaron de tal manera que permitieron hacerles un análisis estadístico. La mayoría de los datos se cuantificó, pero el grueso del análisis es interpretativo. El análisis cualitativo al que nos referimos se realizó con el propósito de descubrir conceptos y relaciones entre los datos recogidos de las diferentes fuentes. Los datos que se recogieron también incluyeron documentos textuales (ensayos escritos por los estudiantes de último semestre) y datos de procedencia estadística como las encuestas y los cuestionarios.

Básicamente, la investigación que aquí se presenta se llevó a cabo con los tres componentes principales existentes en la investigación cualitativa (Strauss A. \& Corbin J., 1990) a saber: Primero, están los datos que provinieron de fuentes diferentes, tales como entrevistas, observaciones, documentos, registros, encuestas y/o cuestionarios. Segundo, están los procedimientos utilizados para analizar, organizar e interpretar los datos. Entre estos, se encuentran: conceptualizar y reducir los datos, elaborar categorías en términos de sus propiedades y dimensiones, y relacionarlos por medio de una serie de oraciones proposicionales. Al proceso de conceptualizar y reducir los datos se le denomina codificación. El tercer componente se refiere a los informes escritos y verbales finales, es decir, los resultados finales. Este método cualitativo permitió como primera medida explorar áreas sustantivas sobre las cuales se conocía poco y segundo, obtener un conocimiento nuevo acerca del fenómeno estudiado (Strauss A. \& Corbin J., 1990).

El enfoque etnográfico en esta investigación permitió penetrar en la concepciones, tradiciones, roles, valores y normas del ambiente en que se vive y se dan las prácticas de lectura y escritura en las licenciaturas. Además, sirvió de gran ayuda para explicar la conducta individual y grupal de la manera como se dan las prácticas de lectura y escritura en estas licenciaturas. La interacción con las partes constituyentes, docentes, estudiantes, textos, contextos, etc. dieron acceso a una nueva realidad de la manera cómo se desarrollan las prácticas lectoras y escritoras en estas licenciaturas con su función y significado permitiendo crear una imagen realista y fiel del grupo estudiado, así como la comprensión del mismo.

\section{RESULTADOS}

\section{Concepciones de estudiantes y docentes de licenciaturas acerca del aprendizaje y la en- señanza de la lectura y escritura de textos académicos}

De acuerdo con los resultados la mayoría de los estudiantes de los programas de las licenciaturas conciben que el aprendizaje de la lectura y la escritura que han tenido durante su carrera universitaria sea muy limitado. Además, la concepción que tienen de la lectura y la escritura la relacionan con la estructura de la malla curricular. Los estudiantes de las licenciaturas atribuyen la deficiencia de su formación lectora y escritora a la ausencia de cátedras y/o asignaturas para el desarrollo de estos procesos así como también al compromiso de formación de estas competencias en los procesos de enseñanza de las mismas.

De igual forma, le atribuyen sus deficiencias a que estos procesos lectores y escritores sólo los 
desarrollan pocas asignaturas. De acuerdo con los resultados, los estudiantes de estos programas piensan que los procesos de lectura y escritura son asumidos en la enseñanza de algunas áreas o asignaturas. Opinan, asimismo, que el tratamiento que se les da a estos procesos es secundario y son de poca importancia para el desarrollo de los contenidos. Admiten que en la mayoría de las asignaturas sólo se dedican al ejercicio físico, la danza, tocar instrumentos, el moldeo de plástico y artesanías y otros elementos, como también al ejercicio, los problemas y los números.

\section{Carencia de lectura y escritura. Asignaturas prácticas}

Los estudiantes admiten que en su programa existen asignaturas en las que muy poco se lee y se escribe. Señalan, de igual manera, que esto es consecuencia de la dedicación casi exclusiva a la realización de problemas y ejercicios matemáticos y que lo que más les interesa a los profesores es la manera cómo realizan estos ejercicios. Además, perciben que en estas carreras los procesos de lectoescritura son algo secundario y que sus docentes muy poco tienen en cuenta estos procesos para el desarrollo de los contenidos. Estas afirmaciones se señalan en el análisis de los registros de las entrevistas realizadas a los estudiantes. En la Tabla 1 los estudiantes expresan su opinión hacia la lectoescritura.

\section{Tabla 1}

Concepciones de los estudiantes acerca del proceso enseñanza de la lectura y la escritura

\begin{tabular}{|l|l|}
\hline \multicolumn{1}{|c|}{ Pregunta } & \multicolumn{1}{c|}{ Respuesta } \\
\hline $\begin{array}{l}\text { ¿Cómo es el proceso de enseñanza y } \\
\text { aprendizaje de la lectura y la escritura } \\
\text { en tu carrera? }\end{array}$ & $\begin{array}{l}\text { EST } \\
\text { "Bueno muy poco leo y escribo en } \\
\text { clases eh, ya que, esto se debe a que } \\
\text { matemática eh más que todo nos } \\
\text { dedicamos es a realizar ejercicios, pro- }\end{array}$ \\
$\begin{array}{l}\text { blemas, todo lo que se debe con, con } \\
\text { las cuestiones de número". }\end{array}$ \\
\begin{tabular}{l} 
EST. \\
"No porque el tema de la lecto-escritura \\
es algo secundario en la carrera en la \\
que ya estoy y no es algo que se tiene \\
muy en cuenta, sólo nos dedicamos a \\
hacer ejercicios y problemas" \\
EST. \\
\hline "La verdad con poca frecuencia ya que \\
lo que más le interesa a los profesores \\
es la manera de como realizamos los \\
ejercicios de cálculo".
\end{tabular} \\
\hline
\end{tabular}

Fuente: Entrevista a estudiantes 
Igual situación se presenta en otros programas de licenciaturas donde los estudiantes afirman que solo se dedican a estudiar los movimientos, motricidad, las manualidades, los diferentes tipos de bailes y danzas pero que muy poco llevan a cabo un proceso detenido y profundo de la lectura de estos temas. Otros estudiantes, sin embargo, señalan que sí leen y escriben muchísimo pero en pentagramas musicales.

\section{Tabla 2}

Programas de licenciaturas que desarrollan procesos de lectura y escritura

\begin{tabular}{|c|c|}
\hline Pregunta & Respuesta \\
\hline $\begin{array}{l}\text { ¿Cómo ha sido el proceso de enseñanza } \\
\text { y aprendizaje de la lectura y la escritura } \\
\text { en tu carrera universitaria? }\end{array}$ & 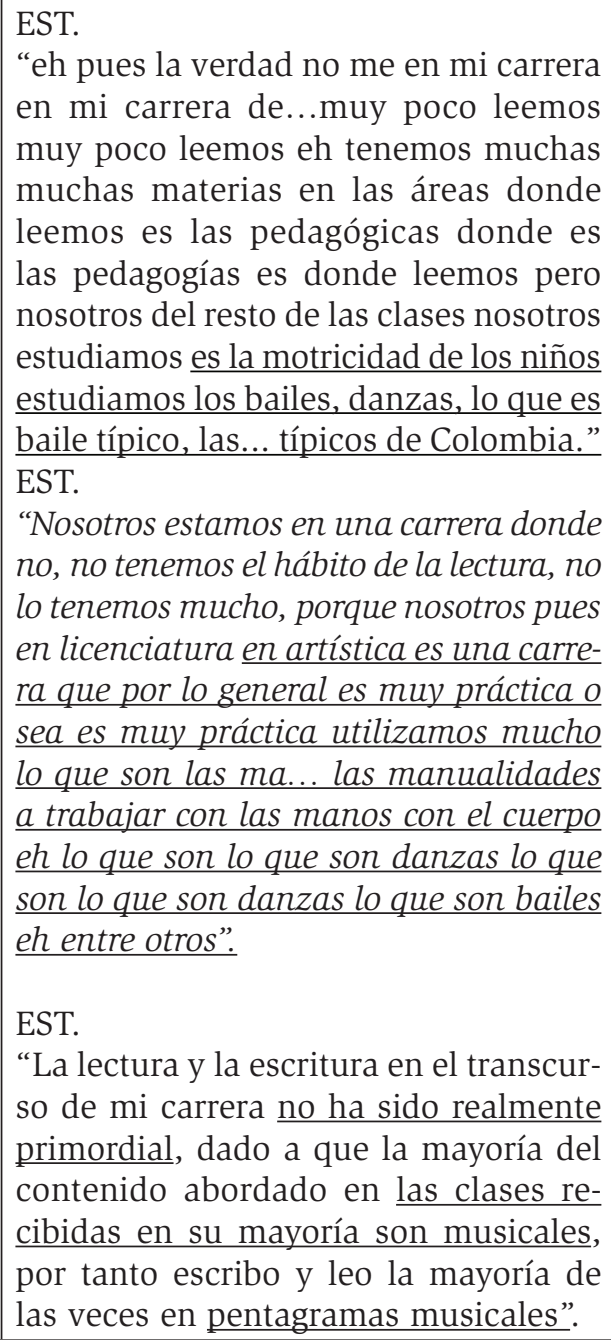 \\
\hline
\end{tabular}

Fuente: Encuesta a estudiantes

Estos resultados (Tabla 2) demuestran que en la mayoría de los programas de licenciaturas hay asignaturas que sólo se dedican a su actividad práctica y no le dan valor a la lectura y la escritura de los contenidos que éstas presentan. Es así como los estudiantes de estos programas han manifestado la debilidad que tienen en estos procesos. 


\section{Poco asesoramiento y/o apoyo en el proceso lector-escritor}

Los estudiantes de las licenciaturas expresan que gran parte de las dificultades en el proceso lectoescritor se debe a que en su programa los profesores les ayudan poco a leer y escribir los textos que se les asignan. Admiten que lo poco que saben lo aprendieron en la institución donde cursaron sus estudios de educación básica. Ellos piensan que esta falta de asistencia en los procesos de lectura y escritura no solo les ha generado dificultades para leer los textos que les asignan sus profesores, también sienten que en consecuencia les cuesta escribir su trabajo de grado.

Llama la atención que los estudiantes entrevistados creen que no se dan estos procesos de lectura y escritura por cuanto los trabajos escritos que presentan a sus profesores (ensayos, resúmenes entre otros escritos), muchas veces no son devueltos con las anotaciones y/o recomendaciones. Por tanto, no saben qué tipo de correcciones hicieron, como tampoco si estaba bien organizado el texto o no. Puntualizan los estudiantes al respecto que en muchas ocasiones cada vez que presentan trabajos escritos a sus profesores, reciben como respuesta que en octavo semestre los ensayos deberían ser excelentes.

(...) y como tenerlo si nunca nos enseñaron o nunca nos corrigieron como deberían hacerse". "no enseñan cómo se debe hacer. "En mi opinión el programa al cual pertenezco no me ha proporcionado las competencias necesarias, considero que las pocas competencias las traigo conmigo desde mi bachillerato, por lo cual se me ha dificultado la redacción de mi proyecto de grado

Fuente: Entrevista a estudiantes

\section{Carencia de los procesos lectores y escritores en los Programas Curriculares y la Malla Curricular (Pensum)}

Los estudiantes de las licenciaturas piensan que la poca formación lectora y escritora que han obtenido durante su carrera es debido a que en las mallas curriculares (pensum) no están incorporadas asignaturas para el desarrollo de estos procesos. Lo que más apunta a determinar la causa principal de estas dificultades que presentan los estudiantes en estas habilidades es la falta de desarrollo de las mismas en las cátedras que cada docente desarrolla en las diferentes disciplinas. En el entorno académico de los programas de licenciaturas se ha prestado poca atención a la función que el lenguaje oral y escrito tiene en la misma construcción de la ciencia y en la construcción del conocimiento en los contextos académicos. De acuerdo con las entrevistas, los estudiantes admiten que tienen poco acompañamiento en los procesos de lectura y escritura para aprender a construir textos en estos entornos. Al parecer, los docentes suponen que los estudiantes ya han adquirido habilidades para construir estos tipos de textos y que lo único que deberán hacer es aplicarlas a nuevos contextos y a nuevos contenidos.

De igual forma, los alumnos señalan que se les enseña poco cómo expresarse hablar científicamente, cómo elaborar frases y párrafos científicos y/o académicos, cómo combinar términos y significados, cómo hablar, argumentar, analizar o escribir en lenguaje científico. Se da por hecho que simplemente van a caer en la cuenta de cómo hacerlo y de los patrones temáticos de la materia. A estos estudiantes de las licenciaturas no se les ayuda a descifrar los códigos que cada disciplina maneja y que se encuentran inmersos en los textos. Las dificultades de lectura y escritura de los estudiantes a menudo no solo se derivan de los procesos defectuosos o ineficaces, sino también de la falta de familiaridad con las convenciones del discurso académico. Al respecto, Johns (1997) señala que la comprensión de la naturaleza de la alfabetización implica saber cómo el conocimiento se representa en las distintas disciplinas y contextos. Además, es estar familiarizado con las estrategias necesarias para comprender y organizar los textos, a sabiendas de los contextos sociales en que los textos se producen y se leen, conocer a la comunidad y la cultura y saber diferenciar ciertos textos y tipos de texto. Por consiguiente, y tal como lo expresan 
los estudiantes de las licenciaturas (Tabla 3), el proceso de formación que reciben en la universidad en cuanto a lectura y escritura es muy poco.

\section{Tabla 3}

Formación lector-escritor

\begin{tabular}{|l|l|}
\hline \multicolumn{1}{|c|}{ Pregunta } & \multicolumn{1}{|c|}{ Respuesta } \\
\hline & $\begin{array}{l}\text { EST. } \\
\text { "Del proceso formativo que me ha ofrecido la univer- } \\
\text { sidad puedo decir que referente al proceso de lectura } \\
\text { y escritura desde el ámbito de mi carrera ha sido un } \\
\text { poco pobre a pesar de las buenas intenciones de algu- } \\
\text { nos profesores en querer implementar metodológicas y } \\
\text { estrategias que promuevan estos dos aspectos tan im- } \\
\text { portantes dentro del campo de la docencia. Considero } \\
\text { que no existe una buena coordinación al momento } \\
\text { crear e implementar en las mallas curriculares las } \\
\text { cuales deben fomentar la lectura y la escritura como } \\
\text { fuentes enriquecedoras de conocimientos, quizás como } \\
\text { estudiantes" } \\
\text { EST. } \\
\text { de formación lector-es- } \\
\text { critor durante tu carrera en la problemática se debe a que en el } \\
\text { universitaria? }\end{array}$ \\
$\begin{array}{l}\text { pensar académico permanentemente debe haber asig- } \\
\text { naturas que propicien estas competencias y que los } \\
\text { docentes aporten acorde a sus capacidades escriturales } \\
\text { nuestro proceso". } \\
\text { EST. } \\
\text { "Yo soy reintegrada y en mi anterior etapa si teníamos } \\
\text { una cátedra de comunicación pero durante solo un } \\
\text { semestre comprendo y es un deber la formación in- } \\
\text { dependiente pero el maestro es un apoyo y no solo el } \\
\text { maestro sino su método, didáctica y plan de trabajo". } \\
\text { EST. } \\
\text { "En el 2007 cursamos asignaturas en las cuales nos } \\
\text { capacitaban para desarrollar y estimular nuestro pro- } \\
\text { ceso lecto escritor, las cuales los semestres siguientes } \\
\text { fueron exiliadas del pensum; }\end{array}$ \\
\hline
\end{tabular}

Fuente: Entrevista a estudiantes

A continuación, se presenta la concepción de ensayo que tienen los estudiantes de las licenciaturas.

Se aplicó una encuesta a estudiantes de últimos semestres de las licenciaturas en la Facultad de Educación de la universidad objeto de estudio. La encuesta estuvo orientada por dos preguntas abiertas acerca de la concepción que tienen de ensayo. Estas fueron:

1. ¿Qué piensas de lo que es un ensayo?

2. ¿De qué manera lo elaboras? 
El número total de estudiantes que respondió fue de 228, todos pertenecientes al último semestre de los diferentes programas de licenciaturas en la Facultad de Educación. La pregunta de la encuesta tuvo como objetivo establecer las concepciones que tienen los estudiantes del ensayo y la manera cómo lo elaboran. A los encuestados se les pidió que escribieran en no más de una cuartilla lo que pensaban del ensayo y como lo elaboraban. Estos fueron los hallazgos:

Concepciones de los estudiantes sobre ensayo y escritura de un ensayo

Tabla 4

Concepción que tienen los estudiantes de licenciaturas acerca del ensayo

\begin{tabular}{|l|c|c|}
\hline Introducción, desarrollo y conclusión & 198 & $86,84 \%$ \\
\hline Ideas principales de un tema, con citas y bibliografía & 166 & $72,80 \%$ \\
\hline
\end{tabular}

Fuente: Información extraída de los resultados de la encuesta 2 aplicada a los estudiantes. Método estadístico descriptivo e inferencial. Hernández y Almeida, 2005; Kerlinger, 1988.

Los resultados de las encuestas que se presentan en la Tabla 4 demuestran que de los 228 estudiantes encuestados, a 198 de ellos (86,84\%), les parece que el ensayo es una especie de resumen de un texto o un tema. Para esta población, el ensayo o resumen, como ellos lo denominan, está compuesto por una introducción, el desarrollo de la idea central o principal y una conclusión. De igual forma, 166 estudiantes, equivalente al 72,80\% de la misma población encuestada, tienen la concepción de que en este escrito (ensayo) se resumen las ideas principales de un tema y que al final se citan los autores que hablan de ese tema.

De acuerdo con los escritos de los estudiantes de las licenciaturas, el concepto de ensayo está relacionado con un breve resumen o un breve texto escrito, en el que para construirlo toman las ideas principales del texto, las agrupan por párrafos y lo convierten en un texto escrito. La concepción formal que tienen para construir el ensayo es que éste debe tener una introducción, un desarrollo y una conclusión. A continuación en la Tabla 5, se presentan algunos escritos de los estudiantes acerca de lo que piensan de la escritura de un ensayo.

La mayoría de los estudiantes opinan que para elaborar un ensayo debe iniciarse con una parte introductoria a partir de las ideas propias acerca de lo que se abordará en el cuerpo del texto y de lo que le parece al estudiante que podría enlazarse con lo que va a ser el desarrollo del ensayo; luego, elaboran una parte final, que entienden como la conclusión de todo, allí se plasmarían la parte del preámbulo y el desarrollo del tema. En la tabla 6 algunas respuestas que dieron los estudiantes de cómo se debe elaborar un ensayo. 


\section{Tabla 5}

Lo que piensan los estudiantes acerca de un Ensayo

\begin{tabular}{|c|c|}
\hline Pregunta & Respuesta \\
\hline ¿Para ti, ¿ qué es un ensayo? & $\begin{array}{l}\text { "Son las ideas que redactamos en pequeños párra- } \\
\text { fos, utilizando las ideas principales de un texto". } \\
\text { "Un ensayo es un escrito en donde tenemos que } \\
\text { plasmar la idea principal o central para argumentar } \\
\text { el texto o temática tratada y leída por el estudiante". } \\
\text { "El ensayo es un escrito breve que trata de cual- } \\
\text { quier tema que se vaya a desarrollar donde se } \\
\text { llevará una idea central del tema y argumentar la } \\
\text { idea propia". } \\
\text { "Un ensayo es el conjunto de ideas principales los } \\
\text { cuales son generadas por una experiencia vivida } \\
\text { de la cual podemos redactar unas palabras para } \\
\text { tratar de resumir esa vivencia". } \\
\text { "Para mí el ensayo es un breve resumen donde } \\
\text { plasman las ideas principales del tema dicho por } \\
\text { autores que hablen acerca de ese tema y que al } \\
\text { final estos autores deben ser citados para hacerle } \\
\text { reconocimiento a ellos de lo que han tratado en } \\
\text { el tema". } \\
\text { "Parte introductoria en donde empiezo a partir de } \\
\text { mis propias ideas de lo de lo que pienso de lo que } \\
\text { me parece que podría enlazarse con lo que va a } \\
\text { ser el desarrollo del ensayo y hago una parte final, } \\
\text { la cual sería la conclusión de todo es que he plas- } \\
\text { mado en la parte del preámbulo y en el desarrollo } \\
\text { del ensayo del tema". } \\
\text { "Pues primero me documento de información que } \\
\text { me sirva para reforzar el texto y lo esencial, em- } \\
\text { pezar por una introducción un desarrollo terminar } \\
\text { con una conclusión". } \\
\text { "Inicio como con una introducción después hago } \\
\text { algo que como un nudo y después como así una } \\
\text { conclusión". } \\
\text { "Lo primero que imagino en mi mente es un texto } \\
\text { con una cantidad de páginas o motor llamadas } \\
\text { cuartillas que colocan el proceso en el cual noso- } \\
\text { tros debemos resumir una temática que sea exigida } \\
\text { por el docente, haciendo o creando una secuencia } \\
\text { que está compuesta por una introducción una idea } \\
\text { central y una conclusión". }\end{array}$ \\
\hline
\end{tabular}

Fuente: Respuestas a la Encuesta aplicada a los estudiantes de Licenciatura sobre el concepto de Ensayo. 


\section{Tabla 6}

Opinión de los estudiantes sobre cómo se debe elaborar un Ensayo.

\begin{tabular}{|l|l|}
\hline \multicolumn{1}{|c|}{ Pregunta } & \multicolumn{1}{|c|}{ Respuesta } \\
\hline Pues primero me documento de informa- \\
ción que me sirva para reforzar el texto y lo \\
esencial, empezar por una introducción un \\
desarrollo terminar con una conclusión". \\
"Inicio como con una introducción después \\
hago algo que como un nudo y después como \\
así una conclusión". \\
"Además, debe tener una introducción de la \\
cual tiene que estar una hipótesis, un desarro- \\
llo en el que se expone o se desenlaza dicha \\
hipótesis y un desenlace o conclusión en la \\
que se da una solución a la hipótesis". \\
"Lo primero que imagino en mi mente es un \\
texto con una cantidad de páginas o motor \\
llamadas cartillas que colocan el proceso en el \\
cual nosotros debemos resumir una temática \\
que sea exigida por el docente, haciendo o \\
creando una secuencia que está compuesta \\
por una introducción una central y una \\
conclusión". \\
"Eh por lo general siempre empiezo haciendo \\
una parte introductoria en donde empiezo a \\
partir de mis propias ideas de lo de lo que \\
pienso de lo que me parece que podría en- \\
lazarse con lo que va a ser el desarrollo del \\
ensayo y hago una parte final, la cual sería \\
la conclusión de todo es que he plasmado en \\
la parte del preámbulo y en el desarrollo del \\
ensayo del tema". \\
Bueno, los ensayos que he hecho, lo primero \\
que hago es con un con una breve introduc- \\
ción quizá muy subjetiva muy particular y \\
después sí lo que hago es cumplir el objetivo \\
eh de dicha temática.
\end{tabular}

Fuente: Encuesta aplicada a los estudiantes

El pensamiento que tienen los estudiantes en general sobre el ensayo es la de un resumen o "un breve escrito" como ellos lo llaman del texto o tema leído. Las respuestas que los estudiantes proporcionan en ambas preguntas permiten comprender que así como ellos tienen el concepto de ensayo, de 
igual forma lo tienen para su elaboración. Esto significa que los estudiantes de licenciaturas tienen concebido un patrón definido de escritura de un ensayo que está constituido por una introducción, el desarrollo de la idea central o principal del tema y una conclusión. Al parecer, los estudiantes de las licenciaturas tienen arraigado este tipo de patrones para la elaboración de un ensayo, ya sea porque así lo han venido desarrollando en todas sus etapas de formación lector-escritor en la universidad o porque en algún momento durante el paso por la escuela adquirieron estos patrones que no se apartan de la composición y estructura de un ensayo académico tipo argumentativo y/o expositivo.

Seguidamente, se dan a conocer las concepciones que tienen los docentes acerca de los procesos de enseñanza y aprendizaje de la lectura y escritura en la universidad. Para el proceso de análisis, interpretación y comprensión de esta categoría se tuvieron en cuenta los resultados que surgieron del análisis de las entrevistas y encuestas realizadas a los docentes. Los elementos (subcategorías emergentes) permitieron una mayor y clara comprensión del concepto que tienen los docentes acerca de la lectura y la escritura en las licenciaturas. A continuación, se describen, analizan e interpretan cada uno de los elementos (subcategorías) que fundamentaron el concepto de la categoría arriba mencionada.

\section{La ortografía y la caligrafía, básicas en la redacción de un texto escrito}

Los docentes de las licenciaturas afirman que la ortografía y la caligrafía son fundamentales para la redacción de un texto escrito. Estos docentes piensan que una buena redacción es aquella que tiene una buena ortografía y caligrafía. La mayoría de los docentes de las licenciaturas admiten que muchos de sus estudiantes, aun estando en niveles superiores, redactan mal, es decir, tienen mala ortografía y caligrafía; asimismo, no saben expresar de manera clara las ideas. A continuación los profesores opinan sobre este tema.

Opinión de los docentes sobre la redacción de los estudiantes
(...) se les evalúa la redacción porque, increíble pero todavía redactan mal o sea ellos creen que las ideas están bien claras y plasmadas, se dan para varias interpretaciones. Algunos redactan pero no llegan, no aterrizan en lo que quieren decir, se les vigila mucho la ortografía, lo mismo en las clases ahi hay muchas fallas, pero como es en lo general, es la redacción

(...) increíble ya casi terminan pero todavía redactan mal con pésima ortografía

(...) los desempeños escritúrales de las estudiantes generalmente son básicos, tienden a confundir un ensayo con un resumen, no poseen en su mayoría visión crítica, poca capacidad para presentar sus propios puntos de vista, tal vez porque llegan a la universidad con falencias en estos procesos que forman parte de las competencias comunicativas. Tienen mucha dificultad para proponer ideas. Ortografía es precaria, al igual que la caligrafía; se les dificulta redactar, no tienen claridad sobre la escritura de un ensayo o la diferencia que existe con otros tipos de textos

Fuente: Entrevista a los docentes

\section{La ortografía y la caligrafía como identidad del docente}

Algunos de los docentes conciben que la ortografía y la caligrafía identifican y califican al docente tanto en lo personal como en lo laboral. Ellos admiten que si un docente no tiene una buena ortografía y una buena caligrafía no podría obtener un buen trabajo.

(...) eeehhh la escritura va por el mismo lado ehh un estudiante que no sea capaz de expresar bien y redactar bien su ideas de forma clara, con una buena ortografía, que con una buena caligrafía, eehh yo digo que su futuro como profesional puede tener muchas dificultades porque hoy en día los psicólogos está a partir de la letra nos quieren analizar y mucha genta es consciente de eso, cuando ya están allá arriba, dicen no cuando yo vaya a buscar mi trabajo al exponer mi firma mi sola firma desdice mucho del profesional que es, o sea yo considero que esto es muy importante 


\section{"Leer y escribir con buena ortografía" Modelos previos de enseñanza y aprendizaje de los docentes acerca de la lectura y la escritura de textos}

Podría decirse que la concepción de algunos docentes entrevistados acerca de la lectura y la escritura se debiera a que recibieron este mismo tipo de enseñanza ya sea en su escolaridad o en la formación universitaria. Se comprende, entonces, que la concepción de enseñanza de la escritura de un texto deviene de la forma como al docente le desarrollaron esta competencia. Léase lo que un docente expresa a este respecto:

Opinión de un docente sobre su propia enseñanza

(...) Pero si por ejemplo tengo tantos años de experiencia, además de cuando me enseñaron a mi que me trataron de enseñar siempre una buena redacción con una buena ortografía yo he tratado con mis estudiantes siempre trabajar alrededor ide! y fuera de eso

Fuente: Texto extraído del proceso de sistematización de ATLAS ti.

Es así como estos docentes experimentan y repiten este tipo de enseñanza y aprendizaje que adquirieron convirtiéndola en un modelo o una didáctica de enseñanza para sus estudiantes.

\section{Carencia de conocimientos previos de los estudiantes acerca de las competencias lectoras y escritoras}

La mayoría de los docentes entrevistados poseen la creencia que sus estudiantes llegan a la universidad con muchas falencias en comprensión lectora y escritura de textos. Ellos piensan que el problema no es de la universidad y que "el problema viene desde atrás". Ellos manifiestan que lo que se hace con los estudiantes en la educación básica va a "redundar" y se va a reflejar en la universidad, por tanto, puntualizan que al estudiante le corresponde y debe prepararse y profundizar en estos procesos antes de que inicie su formación universitaria.

(...) Yo creo que eh el problema no es de la universidad, el problema viene desde atrás, lo que se hace con el estudiante en primaria y en la secundaria va a redundar o sea se va a reflejar en la universidad. Eh... un estudiante que... que... no lea y que no escriba comprensivamente debe prepararse desde antes que llegue a la universidad le corresponde le corresponde eh profundizar ese proceso. (Opinión de un docente universitario)

Las anteriores afirmaciones demuestran que los docentes universitarios siempre están culpando de la mala preparación de los procesos de lectura a la formación inmediatamente anterior recibida por el estudiante. Los docentes suponen que los estudiantes ya deben tener los elementos para leer y escribir.

(...) Los desempeños escriturales de las estudiantes generalmente son básicos, tienden a confundir un ensayo con un resumen, no poseen en su mayoría visión crítica, poca capacidad para presentar sus propios puntos de vista, tal vez porque llegan a la universidad con falencias en estos procesos que forman parte de las competencias comunicativas. Tienen mucha dificultad para proponer ideas. Ortografía es precaria, al igual que la caligrafía; se les dificulta redactar, no tienen claridad sobre la escritura de un ensayo o la diferencia que existe con otros tipos de textos". (Opinión de un docente universitario)

\section{Asignaturas prácticas}

Con relación a este aspecto, algunos docentes manifiestan que la naturaleza de su asignatura es práctica no requieren de procesos de lectura y escritura. Para estos docentes, los procesos de lectura y escritura no son relevantes en la enseñanza y el aprendizaje de la asignatura y que por lo tanto no les hacen falta por cuanto sólo se dedican a realizar ejercicios y problemas matemáticos. De igual forma, docentes de otros programas piensan lo mismo, no hacen énfasis en promover y desarrollar estos procesos lectores y escritores porque su asignatura no lo amerita 
se dedican más que todo a realizar cantos, danza, ejercicios físicos y deportes, como también se dedican al arte o tocar ciertos instrumentos musicales, las manualidades y al desarrollo gráfico etc. Léanse algunas voces de los docentes:

(...) sólo nos limitamos a hacer ejercicios en el tablero

(...)El carácter de mi clase es bastante practico, se busca ofrecer pautas para el desarrollo plástico infantil, teniendo en cuenta las etapas del desarrollo gráfico expresivo del niño; por lo tanto, los procesos lecto-escriturales en las estudiantes son bastante limitados

Para los docentes de algunas licenciaturas el tema de la lectura y la escritura es algo secundario en la carrera. Señalan que en sus programas leen y escriben muy poco en clases. Ellos dicen que se dedican a realizar ejercicios, problemas y todo lo que tiene que ver con las cuestiones de número. Otros docentes también manifiestan que sólo utilizan materiales para realizar manualidades y en otras áreas la mayoría de los contenidos abordados en las clases recibidas en su mayoría es musical. Puntualizan, que la mayoría de las veces leen y escriben en pentagramas musicales

(...) Nosotros estamos en una carrera donde no, no tenemos el hábito de la lectura, no lo tenemos mucho, porque nosotros pues en licenciatura en artística es una carrera que por lo general es muy práctica o sea es muy práctica utilizamos mucho lo que son las manualidades a trabajar con las manos con el cuerpo eh lo que son lo que son danzas lo que son lo que son danzas lo que son bailes eh entre otros.

Estos docentes, tienen la concepción de que los procesos de lectura y escritura no les son indispensables y mucho menos necesarios para su proceso de enseñanza y aprendizaje de la asignatura. Sin embargo, los docentes reconocen y admiten que a pesar de ser una asignatura práctica, los estudiantes requieren de leer para fundamentar sus teorías.

(...)Sin embargo, se necesita leer para fundamentar teóricamente cada tema, para entender los procesos del niño en cada una de las actividades y las relaciones existentes entre la edad y la aplicación de la técnica”.

De acuerdo con los resultados encontrados, son múltiples y diversas las concepciones que tienen los docentes y los estudiantes sobre los procesos de enseñanza y aprendizaje de la lectura y la escritura en la universidad. Estas consideraciones son una alerta para que nosotros los docente de la educación superior revisemos continuamente no solo la forma de transmitir los contenidos sino la forma de aprenderlos, enseñarlos y gestionarlos. Es por ello, que en la educación superior deben venir fuertes vientos de cambio, de nuevas formas de enseñar y aprender Pozo, J.,Scheuer, N., Pérez, M., Mateos, M., Martín, E. y De la Cruz, M. (2006).

La educación ha pasado por muchos modelos de enseñanza y de aprendizaje. De las teorías cognitivas se ha pasado a la orientación constructivista y los enfoques socioculturales. Con estas teorías se ha intentado el mejoramiento de estos procesos en todos los centros escolares desde la educación infantil hasta la educación superior, pero aún quedan en las aulas y prácticas escolares vestigios de aquel conductismo que hace cuatro o cinco décadas dominaba la psicología a los procesos educativos y que todavía perduran en las aulas de clases. Sigue existiendo un conductismo ingenuo larvado en las aulas de clases de la educación en general que se encuentra bajo muchas decisiones o acciones que profesores y alumnos ponen en marcha en su afán de enseñar y aprender. Por ello, la educación actual requiere que los docentes y los estudiantes cambien las representaciones que tienen sobre la enseñanza y el aprendizaje, que tanto docentes como estudiantes cambien las mentalidades o concepciones de las prácticas pedagógicas y, para poder cambiar esas representaciones, es preciso primero conocerlas, saber cuáles son, en qué consisten, cuál es su naturaleza representacional y cuáles sus procesos de cambio y sus relaciones con la propia práctica.

Las concepciones sobre el aprendizaje y la enseñanza, sean estas interpretadas desde cualquier enfoque, son sin duda, antes que nada una 
herencia cultural, un producto de la forma en que en la tradición cultural se organizan las actividades de aprendizaje y enseñanza, en general, de educación y transmisión del conocimiento Manifiestan los autores, que más allá, o más acá de lo que sepamos sobre el aprendizaje y la enseñanza, los profesores y estudiantes tienen creencias o teorías profundamente arraigadas, y tal vez nunca discutidas, sobre lo que es aprender y enseñar, que rigen todas las acciones, al punto de constituir un verdadero currículo oculto que guía, a veces sin nosotros saberlo, la práctica educativa. Sin duda, tanto los profesores como los estudiantes de las licenciaturas tienen sus propias teorías e ideas sobre lo que es aprender y enseñar, pero muchos de ellos no saben que las tienen ni en qué consisten, estas son las llamadas teorías implícitas. POZO, et al., (2006).

Estas teorías o creencias proceden tanto de la instrucción formal recibida sobre los procesos educativos, como de la propia práctica y experiencia diaria que como profesores y estudiantes tienen de su práctica cotidiana con la que de hecho muchas veces colisionan. Para estos autores, la mayoría de los estudiantes ha arraigado las mismas formas de aprender tal y como las han venido haciendo durante su proceso de formación constituyéndolas en su propio currículo oculto que los ha formado sin nunca discutir si verdaderamente es lo pertinente, o sin saber que lo que están o no haciendo lo hacen bien o mal. Esto es lo que hace una práctica repetida día tras día sin nunca ser reflexionada por quienes la realizan. Por tanto, resulta evidente que todos somos producto de unas formas culturales de entender el aprendizaje que están profundamente arraigadas en la mentalidad, pues responden a una tradición de modelos de enseñanza aprendidos. Tanto los docentes como los estudiantes de las licenciaturas enseñan y aprenden en gran medida reproduciendo el modelo que vivieron cuando eran alumnos, de tal suerte que replican las mismas prácticas que han venido realizando durante todo su proceso de formación sin el menor cuestionamiento o discusión. En consecuencia, cambiar las mentalidades de los docentes y los alumnos sobre la enseñanza y el aprendizaje y las formas de promoverlo, requiere conocer los cambios que se están produciendo en la cultura moderna del aprendizaje.

Esta nueva cultura del aprendizaje que emerge se presenta como una nueva forma de relacionarse con el conocimiento que está siendo mediada por los nuevos sistemas de representación, por las tecnologías del conocimiento dominantes en una sociedad. La nueva cultura de la enseñanza y el aprendizaje plantean como objetivo la alfabetización con cada uno de estos sistemas (Internet, páginas Web, informática) que constituyen una nueva exigencia, especialmente para el caso de la educación superior Debe entenderse, que estos nuevos sistemas de aprendizaje no se limitan únicamente a ser un soporte del conocimiento, o un vehículo en el que ese conocimiento se transporta y que, por tanto, hay que saber manejar, sino que estos nuevos sistemas de representación, o tecnologías del conocimiento, han formateado la mente de estos sujetos en esta nueva cultura del aprendizaje, creando en ellos nuevas posibilidades cognitivas, nuevas capacidades o competencias. Cassany y Morales, (2008); POZO, et al., (2006).

Podría decirse, entonces, que cada uno de estos sistemas tecnológicos proporciona un acceso cada vez más fácil y fluido a la cultura del conocimiento así como también permiten el aprendizaje de esta misma cultura. Si bien estamos en la nueva cultura del aprendizaje, también se está ante nuevas formas de relacionarse con el conocimiento y, por tanto, de concebir el aprendizaje. Los estudiantes de las licenciaturas están utilizando cada vez más los sistemas de comunicación tecnológicos, de los cuales la Internet es uno de los que más privilegian para consultar e indagar por la información que se les solicitan. Sin embargo, muchos estudiantes de las licenciaturas siguen abordando los textos con una única función la de reproducir el texto sin cambiarlo ni cambiar su propia memoria haciendo una reproducción literal del mismo texto, directo de él.

Puntualizan al respecto que se hace necesario un papel más activo del lector ante el texto, ligado a las nuevas tecnologías del conocimiento y a sus 
nuevos usos epistémicos (que esas tecnologías hacen posible). Esta nueva revolución tecnológica ahonda en la necesidad de promover lectores activos, que construyan su propio texto a partir de los múltiples y variados textos o fuentes de información que se tienen a disposición. La imprenta hizo posibles muchas formas de leer, y de la misma manera hoy las tecnologías de la información están generando otras nuevas formas de distribuir socialmente el conocimiento, que sin duda hacen necesarias nuevas formas de alfabetización (literaria, gráfica, informática, científica, etc.). Estas nuevas formas de alfabetización están generando una nueva cultura del aprendizaje, a la que la escuela o la universidad no deben dar la espalda y que suponen, por tanto, un nuevo reto para nuestras creencias más profundas sobre la enseñanza y el aprendizaje. POZO, et al., (2006).

En algunos de los señalamientos expuestos por los docentes, se confirma que los estudiantes que ingresan a los estudios superiores carecen de conocimientos específicos sobre la forma de leer y escribir los textos, así como de las categorías de pensamiento que generan las disciplinas en la que se encuentran inmersos. A esto, se suma que muchos de los textos académicos que son utilizados en el salón de clases dan por sabidas las líneas de pensamiento de un campo de estudio y sus polémicas, dejando de lado a los estudiantes, quienes por ser recién llegados las desconocen. La lectura y la escritura de los textos que leen los universitarios podrán ser reconocidos en la medida en que se tome conciencia de dos aspectos fundamentales, a saber: que la lectura y la escritura académicas no son naturales sino propias de las culturas disciplinares, y que son los profesores quienes tienen que ocuparse de su orientación. De esta manera, una disciplina es un espacio discursivo, retórico y conceptual, que está hecha de prácticas discursivas propias, involucradas en su sistema conceptual y metodológico. Por consecuencia, aprender una materia o asignatura no consiste únicamente en adquirir sus nociones y métodos sino en manejar sus modos de leer y escribir característicos. El ingreso de los estudiantes universitarios a una comunidad disciplinar determinada trae consigo la apropiación de los usos instituidos para producir e interpretar sus propios textos, lo cual, sólo puede hacerse con la ayuda de los miembros de esa cultura disciplinar. Carlino, 2005, p.1-3) La lectura y la escritura funcionan como herramientas insustituibles para acceder a las nociones de un campo de estudio para elaborarlas, asimilarlas y apropiarse de ellas. Por lo tanto, ningún docente de ninguna materia debería enseñar sus contenidos desentendiéndose de la manera cómo se aprenden. Una forma de tener en cuenta este aprendizaje es que el docente se ocupe de cómo leen y escriben los estudiantes.

Los estudiantes universitarios necesitan aprender a seguir aprendiendo, por ello requieren de sus docentes no sólo el suministro de los contenidos sino también de los recursos para adquirir los conocimientos de dichos contenidos, en este sentido, utilizarán los recursos en el momento que lo requieran de acuerdo con el contexto y la situación o habrán de aprender en qué casos, para qué, dónde y cómo obtener, interpretar y usar los recursos necesarios para resolver la situación en que se encuentran. Es preciso, por tanto, que el docente incluya explícitamente en el programa real de cada asignatura procedimientos de estudio y estrategias de aprendizaje que fortalezcan las competencias lectoras y escritoras dentro de un campo del conocimiento. (Carlino, 2005, p. 1-3)

Los docentes de las licenciaturas aún creen que aprender y enseñar a leer y a escribir es labor de la educación básica y media. Los estudios sobre alfabetización académica , establecen que los modos de leer y escribir, de buscar, adquirir, elaborar y comunicar conocimiento no son iguales en todos los ámbitos y, por ello, advierte contra la tendencia a considerar que la lectura y la escritura académicas son procesos básicos que se logran de una vez y para siempre antes de la universidad. Esta autora considera que no se puede suponer que un curso de lectura y escritura para un determinado año o tiempo o una determinada asignatura es el único responsable de fomentar estas competencias en los alumnos. La insuficiencia de un seminario o un curso está demostrada, y es reconocida la necesidad de 
ocuparse de la lectura y la elaboración de textos más allá del mero año o tiempo estipulados. La alfabetización académica sólo se logra en un dilatado proceso de aculturación que requiere que los miembros de una comunidad disciplinar se encarguen de guiar a los aprendices hacia sus modos particulares de comprender y producir textos. (Carlino, 2005, p. 1-3)

Por ello, es necesario que los docentes de las licenciaturas se comprometan en la enseñanza de la misma. Los profesores de las distintas cátedras pertenecen en mayor o menor grado a las comunidades disciplinares en calidad de usuarios de la lectura y la escritura en sus áreas del saber; en la mayoría de los casos, no han tomado conciencia de las características de las prácticas discursivas que emplean y disponen de un saber en uso no teorizado. Por tanto, se hace necesario que los docentes se ocupen de enseñar ese saber y para ello necesitan reconstruirlo en un plano conceptual más explícito mediante el trabajo en equipo, en comunidades discursivas con sus colegas de la propia área y de otras que puedan ayudar a hacerlo.

Muchos docentes de las licenciaturas piensan que en la universidad no es conveniente orientar la lectura y la escritura de los estudiantes porque esto fomenta su dependencia. Esta idea se fundamenta en la creencia de que la ayuda brindada a los estudiantes inhibe la independencia y la autorregulación que se espera de ellos en este nivel educativo. Dicen, además, que los estudiantes ya son universitarios y lo suficientemente adultos como para no requerir la guía de los docentes., manifiesta que si bien los universitarios están "maduros" en aquellos aspectos para los cuales su pasado los preparó, no por ello dejan de ser noveles. Ella puntualiza que por haber leído y escrito en los distintos niveles de escolaridad, los alumnos no tienen que saber leer y escribir en otro ámbito distinto. Los estudiantes que ingresan a la universidad no tienen experiencia y son absolutamente novatos en las convenciones de la lectura y la escritura universitarias. Termina sosteniendo que para desarrollar autonomía, los docentes deben enseñar cómo se lee y se escribe en cada una de las asignaturas. (Carlino, 2004, p. 1-7)
De la misma manera, los docentes de las licenciaturas tienen la creencia de que enseñar a leer y a escribir en una asignatura resta tiempo para enseñar los contenidos específicos. Ellos admiten, sin duda, que incluir actividades de lectura y escritura que orienten la comprensión de los contenidos cuesta más tiempo que no hacerlo; este tiempo debe restarse al que habitualmente dedican a exponer esos contenidos. Que el docente exponga un tema no significa que los estudiantes lo incorporen. Las actividades de lectura y escritura incluidas en las asignaturas permiten a los estudiantes elaborar y aprender mejor los contenidos. Ella sostiene que ocuparse de cómo los alumnos leen y escriben los conceptos no quita tiempo en el aprendizaje de éstos aunque sí lo sustraiga a la exposición que realiza el docente. La autora sostiene, además, que incluir actividades de lectura y escritura guiadas en una asignatura hace rendir más el tiempo para que los alumnos comprendan los conceptos, aunque simultáneamente los profesores desarrollen menos contenidos. (Carlino, 2005, p. 1-3)

Por su parte, los estudiantes de las licenciaturas admiten que tampoco existen actualmente en los programas y mallas curriculares procesos para la interacción con la lectura y la escritura que permitan y faciliten el aprendizaje de los contenidos. Por consiguiente, Carlino (2005), admite que los docentes deben planificar las propuestas de lectura y escritura que cada asignatura formula a sus estudiantes; esta es una necesidad que se requiere en el desarrollo de los contenidos que se programen en un curso determinado. Asimismo, dentro de esa planificación deben incluirse como objeto de enseñanza los propósitos de lectura y escritura que la universidad o el programa espera de sus estudiantes. De esta manera, se estará enseñando a leer y a escribir como se hace en las respectivas comunidades discursivas. Al respecto, la misma autora manifiesta que resulta necesario que al planificar las propuestas de lectura y escritura, no sólo se tenga en cuenta qué han de leer los estudiantes, también resulta necesario que los docentes tomen conciencia de los propósitos que cumple la bibliografía elegida según los objetivos de la asignatura, con el fin 
de comunicar a los estudiantes por qué se les solicita que lean y para qué.

\section{CONCLUSIONES}

El proceso de enseñanza y aprendizaje de la lectura y la escritura que se desarrolla en el aula de clases de las licenciaturas es muy limitado y gran parte de las dificultades que presentan los estudiantes en su proceso lector y escritor se deben a que los profesores les ayudan poco a leer y a escribir los textos que se les asignan. Podría decirse, además, que el tratamiento que se les da a los procesos de lectura y escritura de textos en las licenciaturas es precario y que son de poca importancia para el desarrollo de los contenidos. En estos programas muy poco es lo que se lee y se escribe; el interés y propósito de los profesores es la manera cómo realizan los ejercicios. Lo mismo sucede con otro resto de programas de licenciaturas donde sólo se dedican a estudiar los movimientos, la motricidad, las manualidades, los diferentes tipos de bailes y danzas, y donde muy poco se llevan a cabo procesos detenidos y profundos de la lectura de los temas. Por tanto, podemos decir que las dificultades que presentan los estudiantes en estas habilidades es la falta de desarrollo de las mismas en las cátedras que cada docente desarrolla en las diferentes disciplinas. Esto nos indica que se ha prestado poca atención a la función que el lenguaje oral y escrito tiene en la misma construcción de la ciencia y en la construcción del conocimiento en estos entornos académicos.

En cuanto a la concepción que tienen los estudiantes sobre el proceso de elaboración de un ensayo, se concluye que este proceso se ve afectado no tanto por el concepto que tienen los estudiantes sino por el desconocimiento y organización de los elementos que componen y constituyen la construcción de un ensayo. A pesar de esto, los estudiantes de las licenciaturas tienen arraigado un tipo de patrón para la elaboración de un ensayo, que no se aparta de la composición y estructura de un ensayo académico tipo argumentativo y/o expositivo tal y como lo sustentan las teorías (introducción, desarrollo/cuerpo, conclusiones y bibliografía).
Otra de las concepciones que los docentes de las licenciaturas tienen sobre el proceso de enseñanza y aprendizaje de la lectura y la escritura tiene que ver con las características de la asignatura que desarrollan. Con respecto a este aspecto, algunos docentes piensan que por las características de su asignatura que, es bastante práctica, no deben hacer uso de procesos de lectura y escritura. Para estos docentes, estos procesos no son relevantes en la enseñanza y el aprendizaje de la asignatura y, en consecuencia, no les hacen falta por cuanto sólo se dedican a realizar ejercicios y problemas matemáticos. De igual forma, docentes de otros programas piensan lo mismo, no hacen énfasis en la promoción y desarrollo de la lectura y la escritura porque su asignatura no lo amerita, pues se dedican más que todo a organizar cantos, danza, ejercicios físicos y deportes, como también al arte (instrumentos musicales, las manualidades y al desarrollo gráfico, etc.).

\section{REFERENCIAS BIBLIOGRÁFICAS}

Carlino, P. (2005). Escribir, leer y aprender en la Universidad. Una introducción a la alfabetización académica. Fondo de cultura económica de Argentina. (p, 1-3)

Carlino, P. (2004). Escribir y leer en la universidad: responsabilidad compartida entre alumnos, docentes e instituciones. En: Textos en contexto 6. Leer y escribir en la universidad. Asociación Internacional de Lectura/Lectura y Vida. Buenos Aires. (p, 1-7)

Cassany D., \& Morales. (2008) Leer y escribir en la universidad: Hacia la Lectura y la Escritura crítica de géneros científicos Universitat Pompeu Fabra, Departamento de Traducción y Filología, Ramblas 30-32, Barcelona, España.

Cassany, D. (2008). Leer en la red. En: Narváez y Cadena. (2008).Los desafíos de la lectura y la escritura en la educación superior.

Cassany, D. et al. (1998). Enseñar lengua. Editorial Graó, de serveis pedagogics.

Cassany, D. (2006). Tras las líneas. Editorial Anagrama, S.A., Barcelona, España.

Estienne, V. y Carlino, P. (2004). Leer en la universidad: enseñar y aprender una cultura nueva. Buenos Aires, 2004.

Goetz, J. P. y Lecompte. M. D. (1988). Etnografía y diseño cualitativo en investigación educativa. Madrid: Morata.

Hammersley, M. y Atkinson, P. (1994). Etnografía. Métodos de investigación. Editorial Paidós. 
Johns, A. M. (1997). Text, role and context. Developing Academic Literacies. Cambridge University Press.

Kerlinger F. (1988). Investigación del Comportamiento. Tercera Edición. Editorial MacGraw Hill.

Lave J., \& Wenger, E. (1991) .Situated Leraning: Legitimate peripheral participation. Cambridge: CUP

Laville, C.; Dionne, J. A. (1999). Construcao do saber: Manual de metodología da pesquisa em ciencias humanas. Porto Alegre: Artes Médicas; Belo Horizinte: Editora UFMG.

Martínez, Miguel (1997). La investigación cualitativa etnográfica en educación. Bogotá: Círculo.

Parodi, G. (2010). Saber leer. Editorial Aguilar.

Parodi, G. (2008). Géneros académicos y géneros profesionales. Ediciones universitarias de Valparaiso, Chile.
Pozo, J., Scheuer, N., Pérez, M., Mateos, M., Martín, E. y De la Cruz, M. (2006).Nuevas formas de pensar la enseñanza y el aprendizaje. Las concepciones de profesores y alumnos. GRAÓ, Editorial.

Rincón y Gil, (2011). Estado de tendencias en las prácticas de lectura y escritura académica en la universidad del Valle.

Rincón y Gil, (2013). Lectura y escritura académica en la Universidad del Valle. Programa editorial. Cali, Colombia.

Saville-Troike, M. (1989). The ethnography of communication. Blackwell Publishers Ltd. Oxford, UK. Strauss, A., Corbin, J. (1990). Basics of qualitative research. Thousand Oaks: Sage.

Woods, P. (1995). La escuela por dentro. Paidós. Barcelona. 